\title{
NOTE
}

\section{Surface Treatment of Flow Channels in Microfluidic Devices Fabricated by Stereolithography}

\author{
Kanako Ohtani ${ }^{1}$, Masaki Tsuchiya ${ }^{1}$, Hitomi Sugiyama ${ }^{1}$, Toru Katakura ${ }^{2}$, \\ Masatoshi Hayakawa ${ }^{2}$ and Toshimitsu Kanai ${ }^{1 *}$ \\ ${ }^{1}$ Department of Materials Science and Engineering, Yokohama National University (79-5 Tokiwadai, Hodogaya, Yokohama, Kanagawa \\ 240-8501, JAPAN) \\ ${ }^{2}$ Atsugi Tec., Sony Corporation (4-14-1 Asahi, Atsugi, Kanagawa 243-0014, JAPAN)
}

\begin{abstract}
A microfluidic device with three-dimensional flow channels was fabricated by stereolithography, and hydrophilic surface treatment of the flow channel was performed by coating the wall of the channel with a silica layer. After the treatment, the device produced monodisperse oil-in-water $(\mathrm{O} / \mathrm{W})$ emulsions. The silica layer on the channel surface was then coated with a fluorinated silane coupling agent to make it hydrophobic, thus enabling the treated device to produce monodisperse inverted water-in-oil (W/O) emulsions.
\end{abstract}

Key words: microfluidics, emulsions, surface treatment, monodispersity, stereolithography

Microfluidic devices, which consist of networks of channels with micrometer dimensions, have attracted considerable attention in a wide range of applications including analytical systems, biomedical devices, and as tools for chemistry and biochemistry based experiments ${ }^{1-5)}$. These devices can produce monodisperse droplets with exceptional precision, which are useful as individual compartments for chemical reactions and templates for preparation of monodisperse functional particles ${ }^{6-11)}$. To date, several types of microfluidic devices have been developed. For example, capillary microfluidic devices consist of coaxial assemblies of tapered glass capillaries on glass slides ${ }^{12,13)}$. Although the device can be fabricated at low cost, it is cumbersome to set the positions and sizes of the capillaries precisely. There are also polydimethylsiloxane(PDMS) devices, which consist of patterned microchannels in a silicone elastomer of PDMS fabricated by soft lithography techniques $^{14-16)}$. Although using soft lithography facilitates accurate control of the positions and sizes of the channels in the device through the design of mask patterns, it is difficult to fabricate flow channels in three dimensions, which would limit the utility of the device for many applications.

We recently developed microfluidic devices with threedimensional flow channels by stereolithography ${ }^{17}$. Stereolithography is a technique for building a three-dimensional object layer by layer through photopolymerization of a liquid monomer resin on the basis of computer-aided design $(\mathrm{CAD})$ data $^{18)}$. Therefore, fine-tuning of the three-dimensional channels can be easily and efficiently performed. We reported that microfluidic devices with co-flow geometry fabricated by stereolithography could produce monodisperse water-in-oil(W/O) emulsions and the size of the monodisperse water droplets could be controlled by changing the flow rates. Furthermore, we demonstrated that monodisperse thermosensitive poly ( $N$-isopropylacrylamide) (PNIPAM) ${ }^{19)}$ microgel particles, which have potential for use in drug delivery ${ }^{20}$ and actuators for biological applications ${ }^{21)}$, could be prepared by polymerization of a gelation reagent dissolved in the monodisperse water droplets. Although stereolithography is a promising method for fabricating three-dimensional microfluidic devices, the default properties of the resin are inappropriate for many applications. For example, the device cannot produce oilin-water $(\mathrm{O} / \mathrm{W})$ emulsions and double emulsions, consisting of drops of oil and water assembled into a core-shell structure, owing to the hydrophobic surface properties of the flow channels ${ }^{22,23)}$. To broaden the applicability of the devices, control of the wetting characteristics of the surface of channels is necessary.

In this paper, we report on the surface treatment of the

*Correspondence to: Toshimitsu Kanai, Department of Materials Science and Engineering, Yokohama National University, 79-5 Tokiwadai, Hodogaya, Yokohama, Kanagawa 240-8501, Japan

E-mail: tkanai@ynu.ac.jp

Accepted August 30, 2013 (received for review August 9, 2013)

Journal of Oleo Science ISSN 1345-8957 print / ISSN 1347-3352 online

http://www.jstage.jst.go.jp/browse/jos/ http://mc.manusriptcentral.com/jjocs 
flow channel in a microfluidic device fabricated by stereolithography. The hydrophilic surface treatment of the flow channel was performed by coating it with a silica layer. We show that after the silica coating, the device produced monodisperse $\mathrm{O} / \mathrm{W}$ emulsions. The silica layer on the channel surface was then coated with a fluorinated silane coupling agent to make it hydrophobic. We show that after the hydrophobic surface treatment, the device produced monodisperse inverted W/O emulsions again.

We designed a microfluidic device with co-flow geometry on a computer using CAD software (SolidWorks). The device had two coaxially aligned cylindrical channels perpendicular to the substrate, as shown in Figs. 1a-c. The internal diameters of the inner and outer channels were 50 and $1000 \mu \mathrm{m}$, respectively. Port A was set for injecting the inner phase so that it flowed through the inner channel to the outer channel, whereas port B was for injecting the outer phase so that it flowed through the outer channel in the same direction. The outlet was used for collecting the droplets of the inner phase formed at the end of the inner channel together with the outer phase. The CAD data were converted into a rapid prototyping format (STL file) and
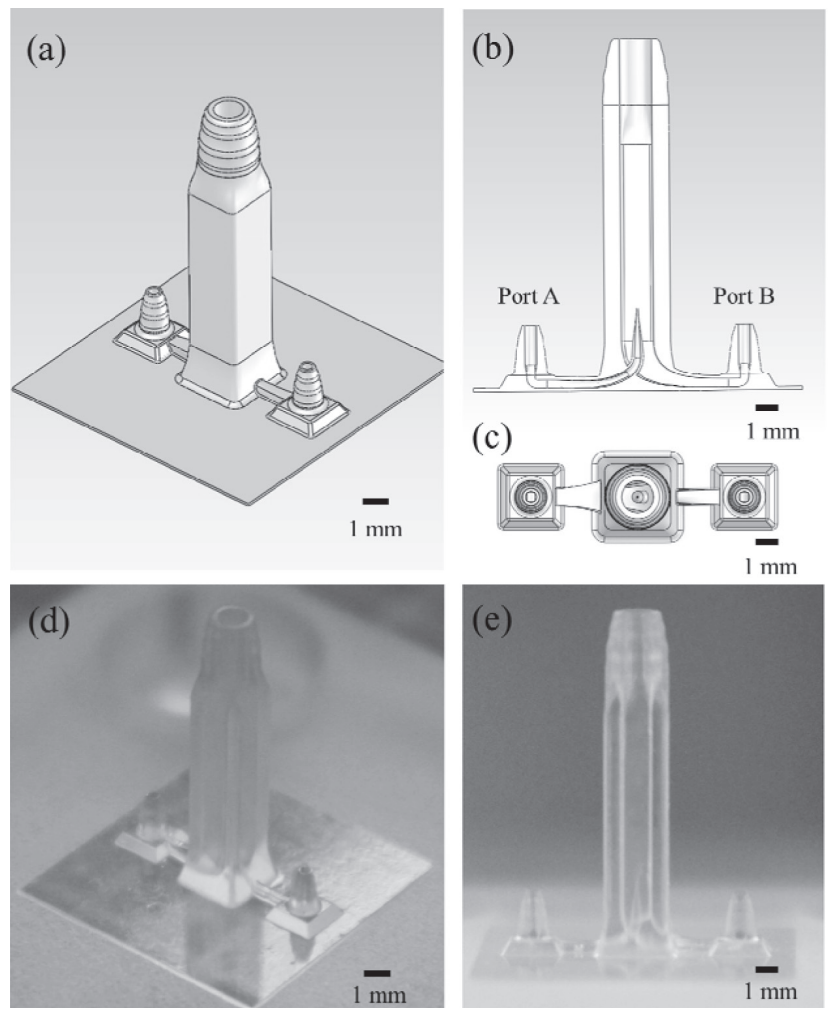

Fig. 1 (a) CAD model of the microfluidic device with co-flow geometry. (b) Side cross-sectional view of the CAD model along the channel axis at the center. (c) Top view of the CAD model. (d) and (e) Photographs of the microfluidic device fabricated by stereolithography. sliced into a set of thin layers, each with a thickness of 3 $\mu \mathrm{m}$. The processing data were then transferred to a stereolithography machine (Sony Corp.). An UV laser beam with a wavelength of $365 \mathrm{~nm}$ was used to scan over a liquid photopolymer epoxy resin (Sony Chemical \& Information Device Corp.) based on the sliced data, and the device was built layer by layer. Figures $1 \mathrm{~d}$ and $\mathrm{e}$ are the photographs of the fabricated device. The three-dimensional structure was fabricated to within an accuracy of less than $5 \mu \mathrm{m}$.

For the preparation of monodisperse $\mathrm{O} / \mathrm{W}$ emulsions, the hydrophilic surface treatment was applied to the inside and outside walls of the outer and inner channels in the device, respectively. A hydrolyzed ethyl silicate solution (N103X, Colcoat Co.) was injected into the outer channel at a flow rate of $10 \mathrm{~mL} / \mathrm{h}$ for $5 \mathrm{~min}$. In order to prevent the coating of the inside wall of the inner channel, air was flowed through the inner channel during the treatment. The device was then put on a $120^{\circ} \mathrm{C}$ hotplate for $30 \mathrm{~min}$ to vaporize the solvent in the channel and cure the coating on the walls. After the hydrophilic treatment, the hydrophobic surface treatment was applied to the silica surface so that the device could produce inverted W/O emulsions again. A fluorinated silane coupling agent (triethoxy- $1 \mathrm{H}, 1 \mathrm{H}, 2 \mathrm{H}$, 2H-tridecafluoro-n-octylsilane, Tokyo Chemical Industry Co.) and air were injected into the outer and inner channels at flow rates of 10 and $50 \mathrm{~mL} / \mathrm{h}$, respectively, for $1 \mathrm{~min}$. In this process, the silanol groups of the agent bond to the silica layer, and the terminal fluorine portions of the agent make the surface hydrophobic.

Figures $2 a$ and $b$ show the SEM images of the inside surface of the inner and outer channels, respectively, in the device after application of the silica coating. A laminated structure, which is artifact of the stereolithography process, was observed on the inside surface of the inner channel. By contrast, for the outer channel, the silica layer covered the surface and the laminated structure was smoothed over. The wettability of the surface before and after the coatings was confirmed by measuring the contact angle of a water droplet on a flat surface made of the same epoxy resin. The uncoated surface had a contact angle of approximately $79^{\circ}$ (Fig. S1a, Supporting information). By contrast, the surface after the silica coating exhibited a hydrophilic contact angle of $33^{\circ}$ (Fig. S1b, Supporting information). After coating the silica surface with the fluorinated silane coupling agent, the water droplet beaded up and the surface exhibited a hydrophobic contact angle of $107^{\circ}$ (Fig. S1c, Supporting information).

The microfluidic device treated with the silica produced monodisperse O/W emulsions, as shown in Fig. 3. Silicone oil (50 cSt, Sigma-Aldrich) and ultrapure water containing a surfactant (3 wt\%, Tween 20) were infused into the inner and outer channels, respectively, by positive displacement syringe pumps. The oil phase flowed through the inner channel to the outer channel while the water phase flowed 

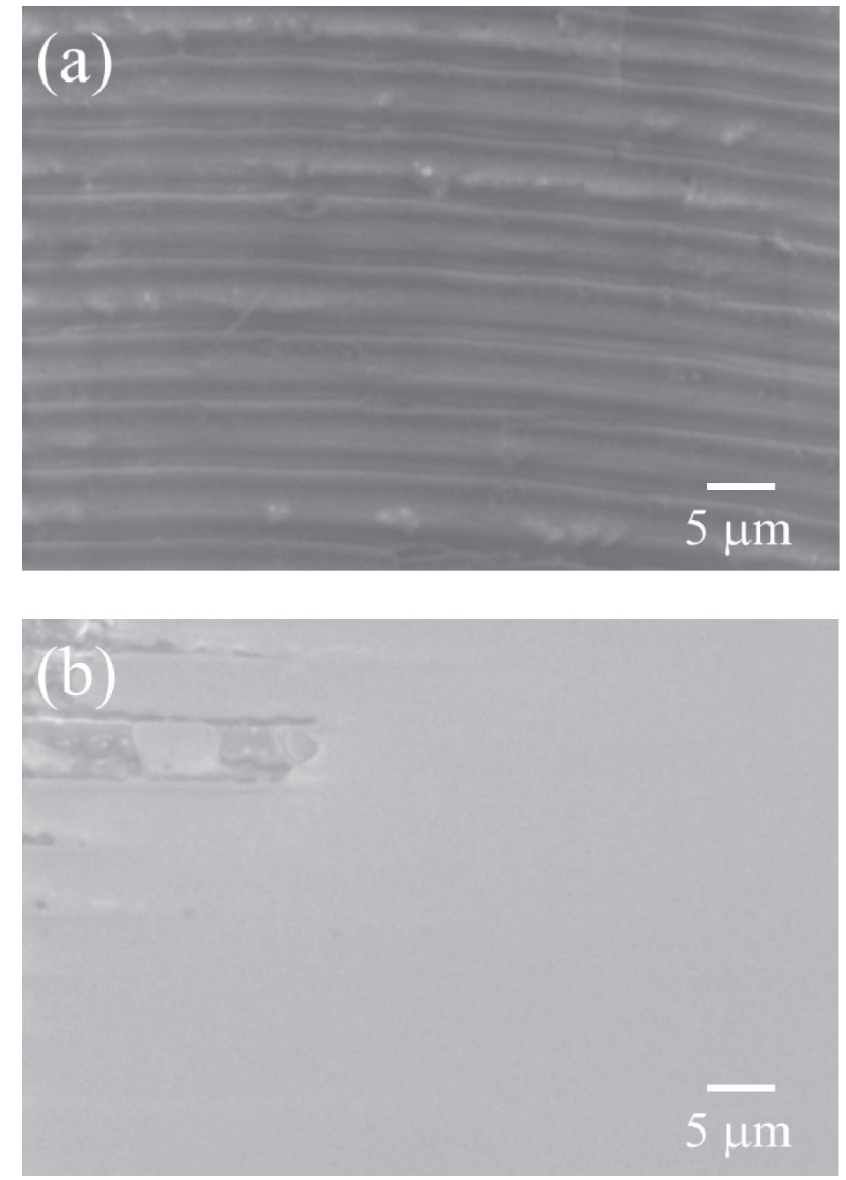

Fig. 2 SEM images of the inside surfaces of the (a) inner and (b) outer channels in device after the coating. The upper-left part of the surface in (b) was scraped on purpose.

through the outer channel in the same direction. At the end of the inner channel, individual monodisperse oil droplets were formed periodically (Fig. 3a). The surfactant molecules added in the water phase immediately migrated to the water-oil interface to stabilize the droplets. Therefore, even after its collection, the emulsion remained stable and monodisperse (Figs. 3b-e). The coefficient of variation (CV) of the droplet size was approximately $2 \%$. As shown in Fig. 3f, the size of the monodisperse droplets could be controlled by changing the flow rates. For example, when the flow rate of the inner oil phase was maintained constant at $0.1 \mathrm{~mL} / \mathrm{h}$, the diameter of the oil droplets decreased from approximately 610 to $290 \mu \mathrm{m}$ monotonically as the flow rate of the outer water phase was increased from 10 to $300 \mathrm{~mL} / \mathrm{h}$.

Upon treatment of the silica surface with fluorinated silane, the device was able to produce monodisperse inverted W/O emulsions again. When ultrapure water and silicone oil containing a surfactant $(1 \mathrm{wt} \%$, Shin-Etsu Chemical Co., KF-6017) were infused into the inner and outer


Fig. 3 (a) Formation of oil droplets in the microfluidic device after the hydrophilic treatment. The flow rates of the inner oil and outer water phases were 0.1 and $5 \mathrm{~mL} / \mathrm{h}$, respectively. Optical micrographs of the monodisperse oil droplets formed as a function of the flow rates of the outer water phase when the flow rate of the inner oil phase is constant at $0.1 \mathrm{~mL} / \mathrm{h}$ : (b) 10 , (c) 50 , (d) 100 , and (e) $300 \mathrm{~mL} / \mathrm{h}$. (f) Plots of the diameter of the oil droplets at a constant flow rate of the inner oil phase as a function of the flow rate of the outer water phase.

channels, respectively, monodisperse W/O emulsions were prepared (Fig. S2, Supporting information). The size of the 
monodisperse water droplets could be controlled by changing the flow rates, similar to the formation of $\mathrm{O} / \mathrm{W}$ emulsions through a hydrophilic device; an increase in the flow rate of the outer phase decreased the size of the droplets monotonically, whereas an increase in the flow rate of the inner phase increased the size of the droplets.

In summary, we have demonstrated the surface treatment of the flow channel in a microfluidic device fabricated by stereolithography. By coating the channel with a silica layer, the device was able to produce monodisperse $\mathrm{O} / \mathrm{W}$ emulsions. By subsequently treating the device with a fluorinated silane coupling agent, the device was able to produce monodisperse inverted W/O emulsions. These results would broaden the utility of such microfluidic devices fabricated by stereolithography.

\section{ACKNOWLEDGMENTS}

This work was supported by the Kazuchika Okura Memorial Foundation and the Kanagawa R\&D Network Plan.

\section{Supporting Information}

This material is available free of charge via the Internet at http://dx.doi.org/jos.63.10.5650/jos.ess13132

\section{References}

1) Whitesides, G. M. The origins and the future of microfluidics. Nature 442, 368-373 (2006).

2) Shah, R. K.; Shum, H. C.; Rowat, A. C.; Lee, D.; Agresti, J. J.; Utada, A. S.; Chu, L. Y.; Kim, J.-W.; FernandezNieves, A.; Martinez, C. J.; Weitz, D. A. Designer emulsions using microfluidics. Mater. Today 11, 18-27 (2008).

3) Pipper, J.; Inoue, M.; Ng, L. F.-P.; Neuzil, P.; Zhang, Y.; Novak, L. Catching bird flu in a droplet. Nat. Med. 13, 1259-1263 (2007).

4) Ahn, K.; Kerbage, C.; Hunt, T. P.; Westervelt, R. M.; Link D. R.; Weitz, D. A. Dielectrophoretic manipulation of drops for high-speed microfluidic sorting devices. Appl. Phys. Lett. 88, 024104-1-024104-3(2006).

5) Pregibon, D. C.; Toner, M.; Doyle, P. S. Multifunctional encoded particles for high-throughput biomolecule analysis. Science 315, 1393-1396 (2007).

6) Teh, S. Y.; Lin, R.; Hung, L. H.; Lee, A. P. Droplet microfluidics. Lab on a Chip 8, 198-220 (2008).

7) Lee, D.; Weitz, D. A. Double emulsion-templated nanoparticle colloidosomes with selective permeability. Adv. Mater. 20, 3498-3503 (2008).

8) Kanai, T.; Lee, D.; Shum, H. C.; Weitz, D. A. Fabrica- tion of tunable spherical colloidal crystals immobilized in soft hydrogels. Small 6, 807-810 (2010).

9) Kanai, T.; Lee, D.; Shum, H. C.; Shah, R. K.; Weitz, D. A. Gel-immobilized colloidal crystal shell with enhanced thermal sensitivity at photonic wavelengths. $A d v . M a-$ ter. 22, 4998-5002 (2010).

10) Kim, J.-W.; Utada, A. S.; Fernández-Nieves, A.; Hu, Z. B.; Weitz, D.A. Fabrication of monodisperse gel shells and functional microgels in microfluidic devices. Angew. Chem. Int. Ed. 46, 1819-1822(2007).

11) Shum, H. C.; Abate, A. R.; Lee, D.; Studart, A. R.; Wang, B.; Chen, C.-H.; Thiele, J.; Shah, R. K.; Krummel, A.; Weitz, D. A. Droplet microfluidics for fabrication of non-spherical particles. Macromol. Rapid Commun. 31, 108-118(2010).

12) Utada, A. S.; Lorenceau, E.; Link, D. R.; Kaplan, P. D.; Stone, H. A.; Weitz, D. A. Monodisperse double emulsions generated from a microcapillary device. Science 308, 537-541 (2005).

13) Chu, L.-Y.; Utada, A. S.; Shah, R. K.; Kim, J.-W.; Weitz, D. A. Controllable monodisperse multiple emulsions. Angew. Chem. Int. Ed. 46, 8970-8974(2007).

14) Whitesides, G. M.; Stroock, A. D. Flexible methods for microfluidics. Phys. Today 54, 42-48(2001).

15) Abate, A. R.; Weitz, D. A. High-order multiple emulsions formed in poly (dimethylsiloxane) microfluidics. Small 5, 2030-2032 (2009).

16) Romanowsky, M. B.; Heymann, M.; Abate, A. R.; Krummel, A. T.; Fraden, S.; Weitz, D. A. Functional patterning of PDMS microfluidic devices using integrated chemo-masks. Lab on a Chip 10, 1521-1524 (2010).

17) Kanai, T.; Ohtani, K.; Fukuyama, M.; Katakura, T.; Hayakawa, M. Preparation of monodisperse PNIPAM gel particles in a microfluidic device fabricated by stereolithography. Polym. J. 43, 987-990 (2011).

18) Rosochowski, A.; Matuszak, A. Rapid tooling: The state of the art. J. Mater. Process. Tech. 106, 191-198 (2000).

19) Saunders, B. R.; Vincent, B. Microgel particles as model colloids: Theory, properties and applications. Adv. Colloid Interface Sci. 80, 1-25(1999).

20) Qiu, Y.; Park, K. Environment-sensitive hydrogels for drug delivery. Adv. Drug Deliver. Rev. 53, 321-339 (2001).

21) Pelah, A.; Jovin, T. M. Polymeric actuators for biological applications. Chem. Phys. Chem. 8, 1757-1760 (2007).

22) Abate, A. R.; Lee, D.; Do, T.; Holtze, C.; Weitz, D. A. Glass coating for PDMS microfluidic channels by solgel methods. Lab on a Chip 8, 516-518 (2008).

23) Abate, A. R.; Krummel, A. T.; Lee, D.; Marquez, M.; Holtze, C.; Weitz, D. A. Photoreactive coating for highcontrast spatial patterning of microfluidic device wettability. Lab Chip 8, 2157-2160 (2008). 